\title{
Development of neutron scattering kernels for cold neutron reflector materials
}

\author{
José Rolando Granada 国, José Ignacio Márquez Damián ${ }^{\natural}$, Javier Dawidowski四,

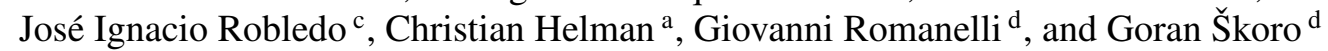 \\ ${ }^{a}$ Departamento Física de Neutrones, Centro Atómico Bariloche, Comisión Nacional de Energía Atómica, RN, \\ Argentina \\ E-mail: granada@cnea.gov.ar \\ ${ }^{\mathrm{b}}$ Spallation Physics Group, European Spallation Source ERIC, Lund, Sweden \\ ${ }^{\mathrm{C}}$ CONICET, Argentina \\ ${ }^{\mathrm{d}}$ ISIS Facility, Rutherford Appleton Laboratory, Chilton, Didcot, Oxfordshire OX11 OQX,U. K.
}

\begin{abstract}
.
The newest neutron scattering applications are highly intensity-limited techniques that demand reducing the neutron losses between source and detectors. In addition, the nuclear industry demands more accurate data and procedures for the design and optimization of advanced fission reactors, especially for the treatment of fuel and moderator materials. To meet these demands, it is necessary to improve the existing calculation tools, through the generation of better models that describe the interaction of neutrons with the systems of interest. The Neutron Physics Department at Centro Atomico Bariloche (CNEA, Argentina) has been developing over the time new models for the interaction of slow neutrons with materials, to produce scattering kernels and cross section data in the thermal and cold neutron energy region. Besides the studies carried out on neutron moderators, we have recently begun looking at materials that could serve as efficient neutron reflectors over those energy ranges. In this work we present the results of transmission and scattering experiments on diamond nanopowder and magnesium hydride, carried out simultaneously at the VESUVIO spectrometer (ISIS, UK), and compare them with newly generated cross-section libraries.
\end{abstract}

Keywords: Nano-diamond, Neutron reflection material, Below cold neutron energy, Total neutron cross section

\section{Introduction}

The tremendous potential of neutrons as a probe of matter or as a research object by itself is limited by the relatively low-intensity flux of neutron sources, as compared with photon sources. In addition, the different processes (production, slowing-down, thermalization) and devices (moderators, transport systems, collimators, energy selectors, detectors, etc.) reduce by several orders of magnitude the actual neutron intensity that eventually conveys the experimental information of interest. For this reason, all optimization tasks that can be carried out on the devices involved are particularly relevant. This, in turn, motivates the creation of new and more precise models of neutron intraction with the systems of interest. The Neutron Physics Department at Centro Atomico Bariloche (CNEA, Argentina) has been developing new models for the interaction of slow neutrons with materials, particularly those of interest for thermal and cold neutron sources. Our aim is to produce scattering kernels and cross section data for the corresponding energy range. The approach involved the determination of the excitation frequency spectra for liquid and solid materials, employing Molecular Dynamics and ab initio calculations, in combination with processing codes (NJOY, NCrystal). Recently we initiated a research line oriented to the search for efficient reflector materials, that may improve the efficiency of guiding surfaces or the actual reflection of neutrons on a containment

${ }^{*}$ Corresponding author. E-mail: granada@cnea.gov.ar 
wall to reduce leakage. A large body of work has been done in the past on that quest, particularly concerning the interaction of slow neutrons with diamond nanoparticles [1]. The high reflectivity of this material for has been demostrated for ultra-cold neutrons (UCN) and very-cold neutrons (VCN) based on calculations for ideal systems as well as by scattering experiments, proposing that such capacity may extend to higher neutron energies, thus bridging the "reflectivity gap" in the neutron spectrum [2]. In a recent work we presented a new scattering kernel based on the combination of bulk diamond particles and coherent interference effects due to the nanostructure [3]. With those ingredients we generated a cross section library over an extended neutron energy range for a real nanodiamond (ND) powder. Reflectivity calculations using our library confirm the excellent performance of that material for UCN and VCN, as well as its rapid decrease for neutron velocities larger than about $150 \mathrm{~m} / \mathrm{s}$.

The reduction of reflectivity in the nanopowder is of course related to the disappearance of the interference contribution to the scattering process as the neutron wavelength becomes shorter than the particle's dimension. Therefore, a different approach was employed to explore the characteristics of potential cold neutron reflectors, based on the requirement of a large scattering cross section combined with a small value of the average cosine of the scattering angle over the energy range of interest $\left(E_{n} \geqslant 10^{-4} \mathrm{eV}\right)$. Those conditions led us to consider metal hydrides, and eventually select magnesium hydride as a good candidate to fulfill those requirements [3]. We have also recently produced a refined scattering kernel for $\mathrm{H}$ in $\mathrm{MgH}_{2}$, [3] and compared the calculated total cross sections with available experimental data at room temperature, obtaining an encouraging agreement. We considered it necessary at this stage to perform specific measurements to experimentally validate the predictions previously stated. In this respect VESUVIO is an almost ideal instrument to simultaneously determine the transmission and scattering properties of diamond nanoparticles and magnesium hydride. In this work we present the results of experiments on diamond nanopowder and magnesium hydride, and compare them with the newly generated crosssection libraries.

\section{Scattering kernels}

\subsection{Scattering kernel for diamond nanoparticles}

Studies on diamond nanopowder have been carried out by Nesvizhevsky and coworkers for quite a long time, involving calculations and measurements on that material to support its use as an excellent reflector for ultraand very-cold neutrons [4] and good quasi-specular reflector for cold neutrons [5]. As the main interest in those studies was the understanding and description of the dominant (elastic) interference effects caused by the scattering particles, those calculations were done for ideal ND systems using the first Born approximation [6]. Recently, SANS and transmission measurements were performed [7], producing the first experimental total cross section results for a diamond nanopowder over the cold neutron energy region.

For the development of our model we consider a system of nanospheres made of bulk diamond. The scattering kernel will involve contributions due to the elastic and inelastic scattering by the diamond lattice, and also the coherent elastic contribution arising from the diffraction of the neutron wave caused by the scattering nanoparticles. The phonon density of states (PDOS) of diamond was evaluated by Density Functional Theory techniques (DFT)[8], showing excellent agreement with the experimental results when used to calculate the specific heat [9]. We then employed the code NJOY [10] for the inelastic cross sections and NCRYSTAL [11] for the elastic ones.

We used a structure factor expression built from the unified exponential/power-law approximation [12] to represent the interference of the neutron-wave caused by the finite size of the (spherical) scattering potential. The structure factor can be integrated to obtain the total scattering cross section due to such interference effects, which presents a $1 / E$ behavior for neutron energies above $\approx 2 \cdot 10^{-5} \mathrm{eV}$. Assuming perfect spherical diamond particles, a simple expression can be derived from the results of the first Born approximation [6]:

$$
\sigma(E)=0.006872 \frac{R^{4.015}}{E},
$$




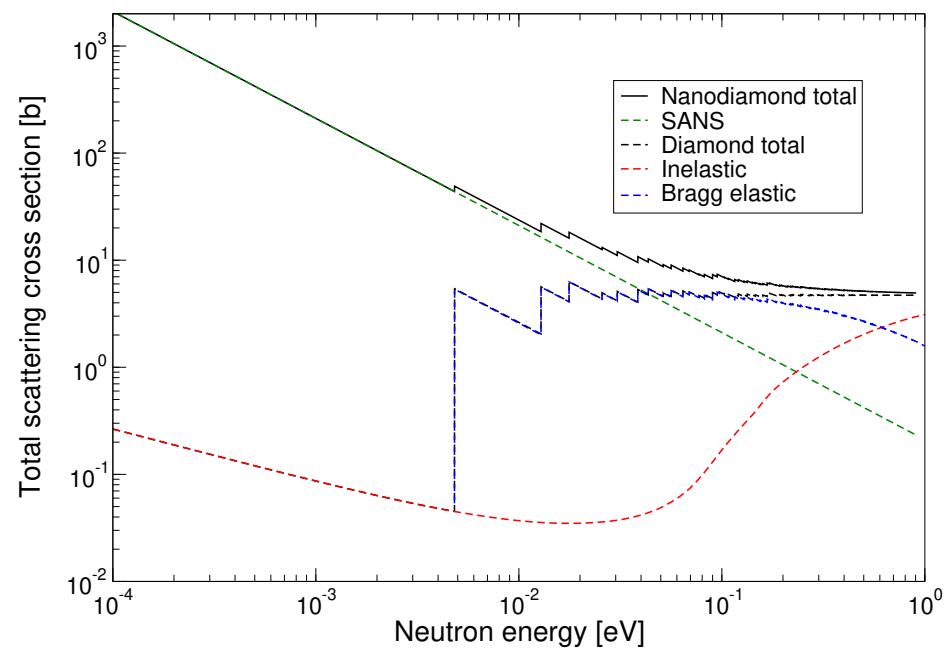

Fig. 1. Total scattering cross section for diamond nanoparticle powder. See text for details.

where $R$ is the average diamond sphere's radius in $\mathrm{nm}, E$ the neutron energy in $\mathrm{eV}$, and $\sigma$ the total coherent cross section in barns. The above formula is accurate enough to determine a reliable value of $R$ from a total cross section measurement. The calculated total cross section of nanodiamond powder at room temperature with $R=2.5 \mathrm{~nm}$ is shown in Fig. 1. where the different contributions of the crystal and SANS are indicated.

\subsection{Scattering kernel for magnesium hydride}

We have proposed recently [3] that $\mathrm{MgH}_{2}$ could be a very interesting material as reflector for cold neutrons, taking advantage of some unique features of the hydrogen in this system: large scattering cross section, high number density, practically no translational motion within the metallic lattice, and unpopulated (acoustic and optical) modes at low temperatures. The latter dynamical characteristics imply a very large effective mass and therefore almost isotropic scattering, and a very small upscattering probability, respectively. We produced a refined scattering kernel for $\mathrm{H}$ in $\mathrm{MgH}_{2}$, [3] and compared the calculated total cross sections with available experimental data at room temperature, obtaining very good agreement. The elastic and inelastic (up and down) scattering components of the total cross section of $\mathrm{H}$ in $\mathrm{MgH}_{2}$ at $20 \mathrm{~K}$ are displayed in Fig. 2. Note that elastic processes dominate the interaction for cold neutrons. This behaviour is due to the absence of low-energy excitation modes that causes the inelastic down-scattering processes to be reduced rapidly below thermal neutron energies.

\section{Experimental details}

The experiments were performed at the VESUVIO spectrometer (ISIS Pulsed Neutron and Muon Source at the Rutherford Appleton Laboratory, United Kingdom). The instument is placed at Target Station TS1. This is a versatile instrument that was originally designed for Deep Inelastic Neutron Scattering and has proven to be very suitable for the measurement of transmissions [13]. The sample-source flight-path is about $11 \mathrm{~m}$. The detection system consists of scattering and transmission detectors. The former are placed in forward and backward scattering directions. In the forward-scattering directions the detector banks are alternated in two characteristic flight-path lengths of about 0.53 and $0.72 \mathrm{~m}$ from the sample position, covering an angular range from $30^{\circ}$ to $70^{\circ}$, whereas in the backward scattering directions the detectors are arranged in three plane panels with sample-detector distances ranging from $0.44 \mathrm{~m}$ (for those placed at the inner positions) to $0.65 \mathrm{~m}$ (for those placed at the peripheral positions), 


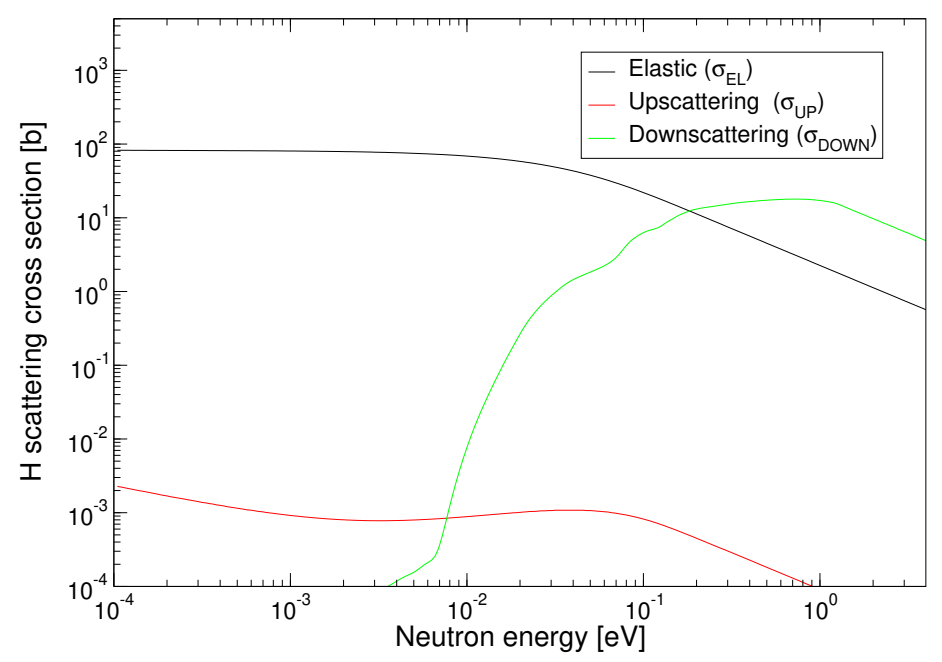

Fig. 2. Comparison of the scattering components for $\mathrm{MgH}_{2}$ at $20 \mathrm{~K}$. $\sigma_{\mathrm{EL}}$ is the elastic component, and the inelastic part is separated into up-scatter $\left(\sigma_{\mathrm{UP}}\right)$ and down-scatter $\left(\sigma_{\mathrm{DOWN}}\right)$.

with angles from $131^{\circ}$ to $166^{\circ}$. The forward scattering detectors are labelled S135 to S198, and the backscattering ones S3 to S134. A schematic representation of the instrument and additional pieces of information are available in Ref. [14].

For the transmission measurements a detector labeled S2 is placed at $13.43 \mathrm{~m}$ from the neutron moderator. A beam monitor $\mathrm{S} 1$ is exposed at the direct beam, at $8.60 \mathrm{~m}$ from the moderator, and its count rate is employed to normalise the spectra registered by the transmission monitor. Both detectors are ${ }^{6} \mathrm{Li}$-doped glass scintillators.

The samples employed in this work were Carbon Nanodiamond and Magnesium Hydride powders. Nanodiamond powder was manufactured by Ray Techniques Ltd. (Israel). It was produced from detonation synthesis and purified. It presents the aspect of a gray powder with a nominal bulk density of $2.7 \mathrm{~g} / \mathrm{cm}^{3}$ with an average crystalline size of $4.3 \mathrm{~nm}$. Metal impurities were not detected and chlorine impurities were less than $1 \mathrm{wt} \%$. The magnesium hydride sample under study was a white micro powder $99 \%$ purity produced by Nanoshel company (USA). The average particle size was 40 to $60 \mu \mathrm{m}$, and its bulk nominal density $1.45 \mathrm{~g} / \mathrm{cm}^{3}$.

The samples were placed into aluminum flat cells of square section $64 \mathrm{~mm}$ inner side and $90 \mathrm{~mm}$ outer side. For Magnesium Hydride a $4 \mathrm{~mm}$ thick cell was chosen, while for Carbon Nano Diamonds thicknesses of $4 \mathrm{~mm}$ and 7 $\mathrm{mm}$ were used. Samples were placed into a standard closed cycle refrigerator (CCR), which allowed to control the temperature during measurements.

\section{Results}

\subsection{Data processing and results for diamond nanoparticles}

Transmitted spectra with and without the sample were processed according to [15], using the S1 monitor for normalization. The data processed directly from the transmission measurements using the Beer-Lambert law show a significant departure from the theoretical model, which suggests that a non-negligible number of the scattering events are counted due to small angle scattering.

To take this effect into account, we performed a simulation of the instrument in Monte Carlo. Geometry was adapted from [16], and consists on a slab source in the position of the moderator $(1101 \mathrm{~cm}$ from the sample position), and two octogonal collimators at 930 and $135 \mathrm{~cm}$ from the sample position, resulting in a circular beam 
with $4.3 \mathrm{~cm}$ diameter in the sample position. The transmission detector was modelled as a $3.5 \times 5.6 \times 1 \mathrm{~cm}^{3}$ slab $244 \mathrm{~cm}$ downstream from the sample. Fig. 3 shows the flux distribution along the midplane of the instrument, superimposed to the geometry. Scalar fluxes are normalized to one source neutron.

Using this Monte Carlo model the contribution of small angle scattering to the detector count was computed, and applied as a correction for sample-dependent background:

$$
\sigma(E)=-\frac{1}{N \Delta x} \log \frac{S-I}{E}
$$

where $N$ is the number density of atoms in the sample, $\Delta x$ the sample thickness, $S$ and $E$ the normalized count for sample and empty container, and $I$ the normalized count for in-scattering events.

Fig. 4 shows different experimental and theoretical results compared. Blue circles represent the experimental total cross section of the $4 \mathrm{~mm}$ thick ND sample computed with the Beer-Lambert law without taking into account in-scattering. When this correction is applied we obtain the data represented by the red circles. A good agreement is found between them and the above mentioned theoretical model (full black line) elaborated by employing the NJOY code for the inelastic cross sections, NCRYSTAL for the elastic ones and the SANS component described in Eq. (1) computed with the value $R=2.4 \mathrm{~nm}$. Also there is agreement with the experimental results of Teshigawara et al [4] (green cicles). In Fig. 4] we also show the total cross section calculated from the spectra determined by Monte Carlo simulations on the experimental setup (dashed black line), which are in agreement with the blue circles. To highlight the importance of the result, Fig. 5 shows separately the corrected experimental total cross section of our nanodiamond sample compared with our model calculations.

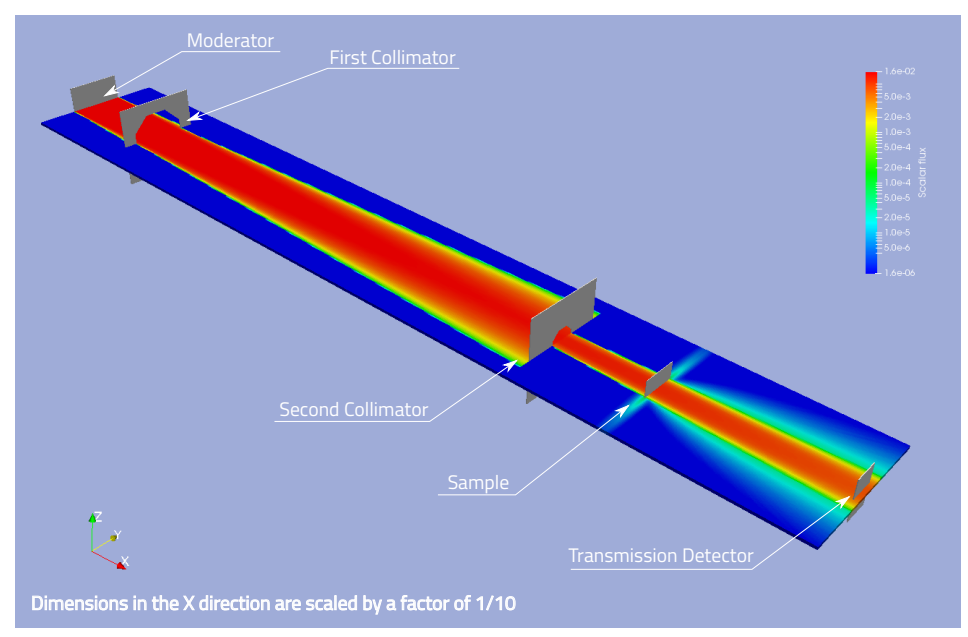

Fig. 3. Flux map computed with the Monte Carlo model. The intensity is normalized to one source particle.

\subsection{Data processing and results for magnesium hydride}

Transmitted spectra with and without the different $\mathrm{MgH}_{2}$ samples were also processed according to [15] using the $\mathrm{S} 1$ monitor for normalization. Data were processed using the Beer-Lambert equation and the total cross section was obtained as

$$
\sigma_{T}(E)=-\frac{1}{N \Delta x} \ln \left(\frac{I_{t}(E, x)}{I_{0}(E)}\right)
$$




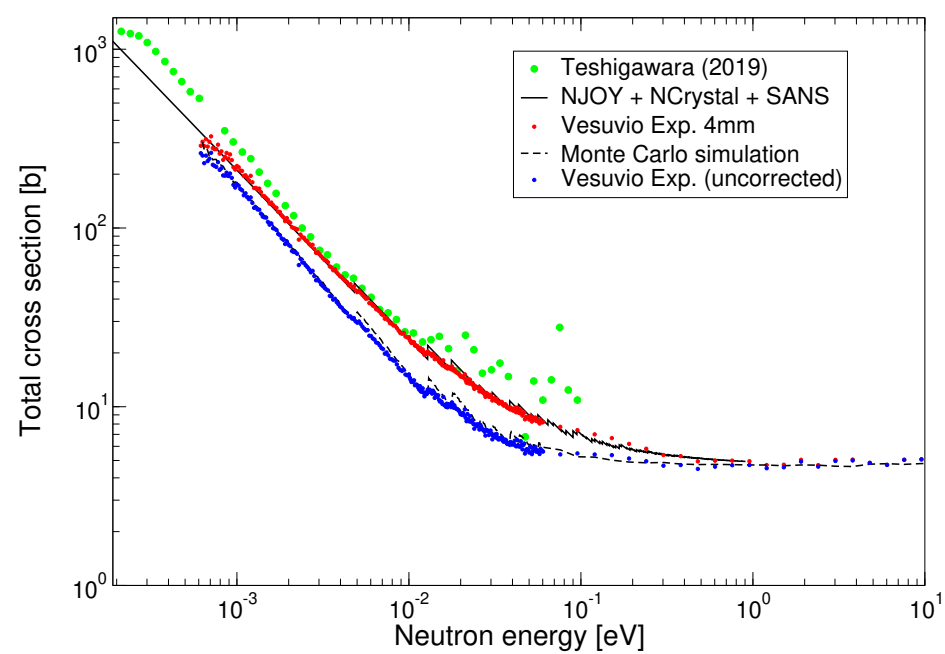

Fig. 4. Experimental total scattering cross section for diamond nanoparticle powder. Red circles show the results computed after the in-scattering correction is applied, and the blue circles were computed with the Beer-Lambert law without taking into account in-scattering. Green circles are previous measurements by Teshigawara et. al [7]. In full line we show the calculated total cross section employing the code NJOY for the inelastic cross sections, NCRYSTAL for the elastic ones and the SANS component described in Eq. (1), while in dotted line the total cross section was calculated from the spectra determined by Monte Carlo simulations on the experimental setup.

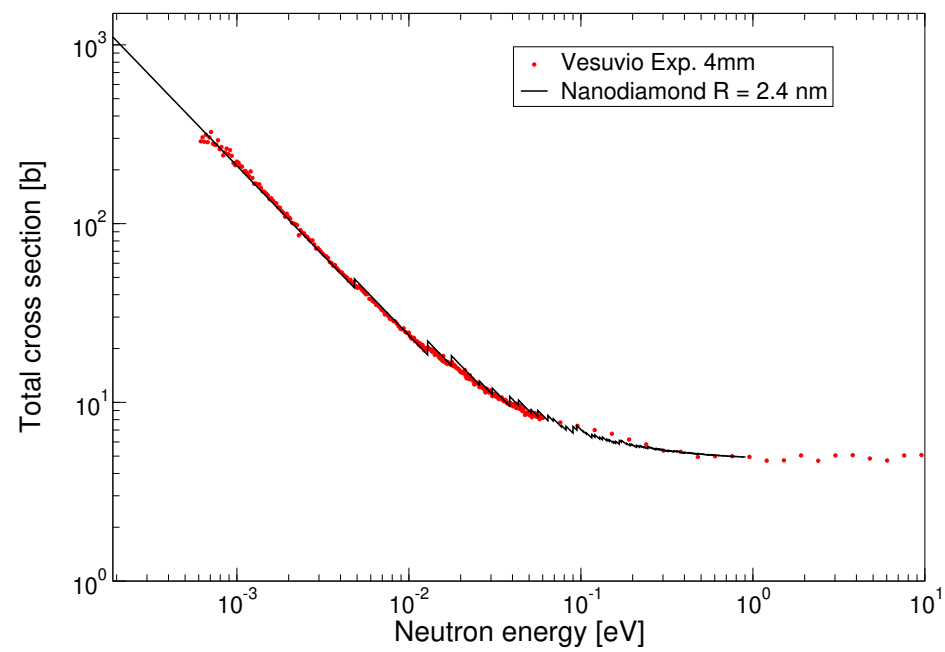

Fig. 5. Corrected experimental cross section compared with our model calculations for an average nanodiamond sphere radius of 2.4 nm.

where $I_{t}(E, x)$ is the transmitted intensity through a sample of thickness $x$ and number density $n$ (number of atoms per unit volume in the sample), and $I_{0}(E)$ is the incident intensity of neutrons with energy $E$. Two $\mathrm{MgH}_{2}$ samples were measured at different temperatures, one at room temperature and another one at $20 \mathrm{~K}$. The resulting total cross sections are displayed in fig.6 As expected, the theoretical model is in good agreement with the experimental results without any extra corrections. This is in agreement with the usual situation in VESUVIO, where for samples without an important small-angle scattering cross section, the signal from scattered neutrons is negligible in the transmission detector [17]. 


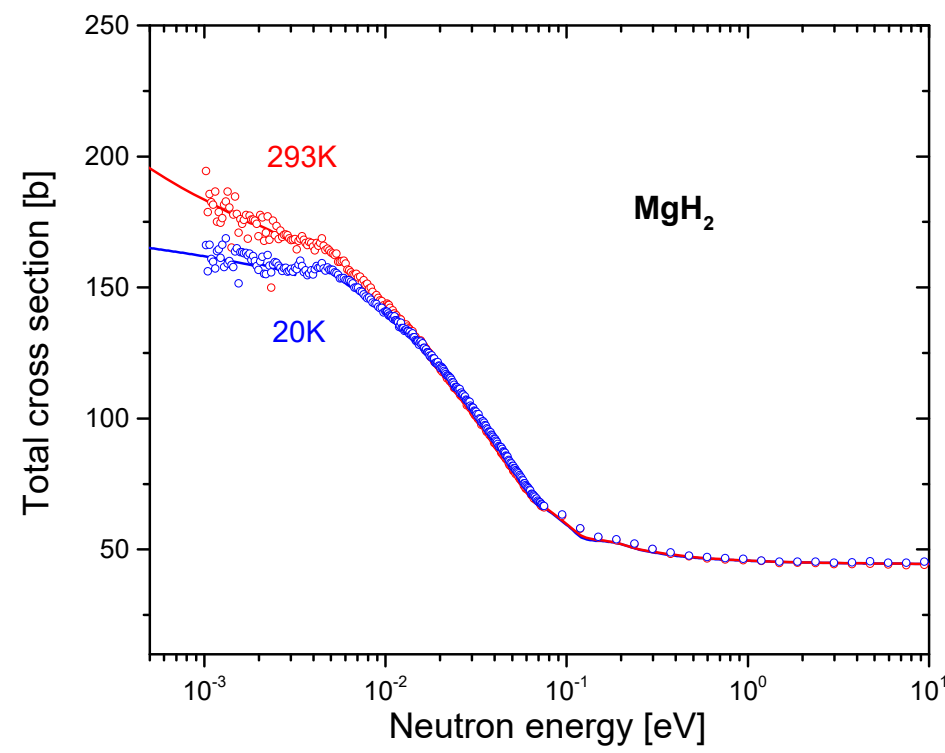

Fig. 6. Experimental total scattering cross section for $\mathrm{MgH}_{2}$ at two temperatures, 20K (blue) and 293K (red). Theoretical models are plotted in continuous lines with their respective colors.

\subsection{Comparing reflectivities}

The relationship stated between the number of neutron counts with energy $E$ of a detector set at an angle $\theta$ for sample $i\left(C^{i}(\theta, E)\right)$ and its reflectivity $R^{i}(\theta, E)$ is

$$
C^{i}(\theta)=\phi(E)\left(1-\operatorname{Tr}^{i}(E)\right) R^{i}(\theta, E) \varepsilon(E),
$$

where $\phi(E)$ is the incident flux, $\varepsilon(E)$ is the detector efficiency, and $\left(1-\operatorname{Tr}^{i}(E)\right)$ the fraction of neutrons not transmitted through the sample. In order to compare the reflective power of both compounds, a ratio proportional to the ratio of their reflectivities was calculated for each detector as

$$
\frac{R^{M g H_{2}}(\theta, E)}{R^{N D}(\theta, E)}=\frac{\left(1-\operatorname{Tr}^{N D}(E)\right)}{\left(1-\operatorname{Tr}^{M g H_{2}}(E)\right)} \frac{C^{M g H_{2}}(\theta, E)}{C^{N D}(\theta, E)},
$$

where $R^{i}(\theta, E)$ denotes a quantity proportional to the reflectivity of the $i^{\text {th }}$ compound (ND refers to nanodiamond) at an angle $\theta$ for neutrons with energy $E$, and $C^{i}(\theta, E)$ the corresponding counts of the detector set at an angle $\theta$ with respect to the incident beam. To obtain $C^{i}(\theta, E)$, the background contribution was subtracted to each detector signal by measuring an empty can sample, and a normalization was performed to the incident beam intensity. The ratio was performed channel by channel using the Mantid framework [18]. The resulting ratio of reflectivities averaged through the detectors taking into account equation (5) is shown in Fig. 7 . 


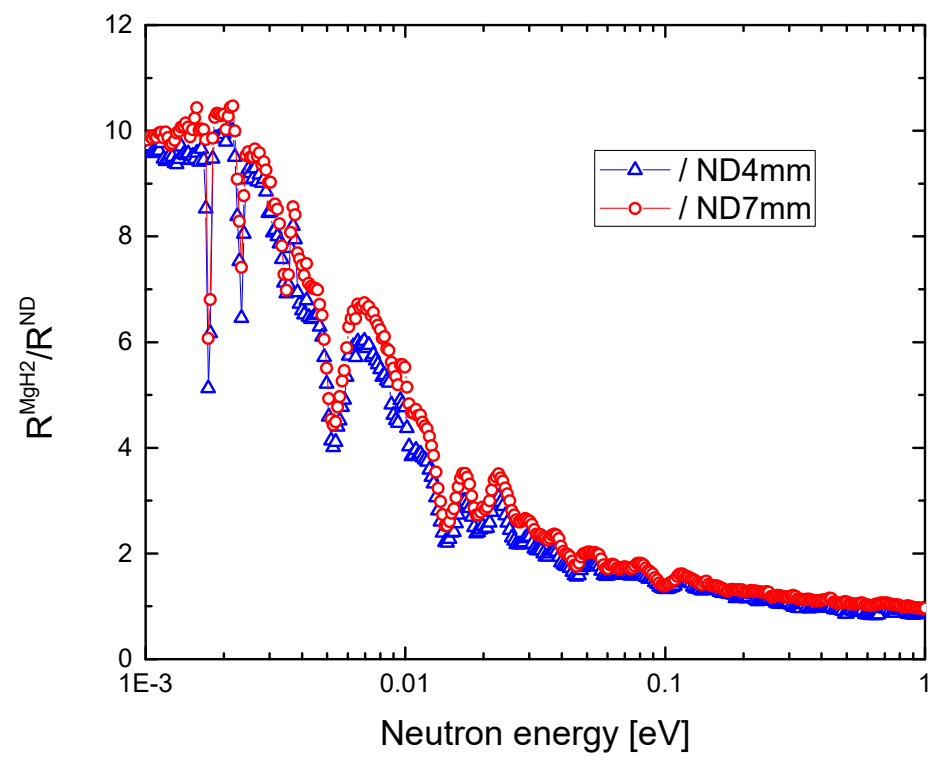

Fig. 7. Energy distribution of the ratio of reflectivities between $\mathrm{MgH}_{2}$ at $20 \mathrm{~K}$ and the nanodiamond sample of $4 \mathrm{~mm}$ (blue) and $7 \mathrm{~mm}$ (red) thickness.

The peak structure seen in Fig. 7 between 1 and $100 \mathrm{meV}$ is due to the presence of sharp Bragg peaks in the nanodiamond sample. Despite this structure, it is interesting to note that the ratio of reflectivities as defined in this work is $\mathrm{R}^{\mathrm{MgH} 2} / \mathrm{R}^{N D} \geqslant 1$ for all energy values below $1000 \mathrm{meV}$, implying that $\mathrm{MgH}_{2}$ is a more efficient reflector than nanodiamonds for thermal and cold neutrons. As to emphasize the angular dependency, a 2D map of the angle and energy distribution of the ratio of reflectivities is shown in Fig. 8, where the displacement of Bragg peaks with the measuring angle is apparent.

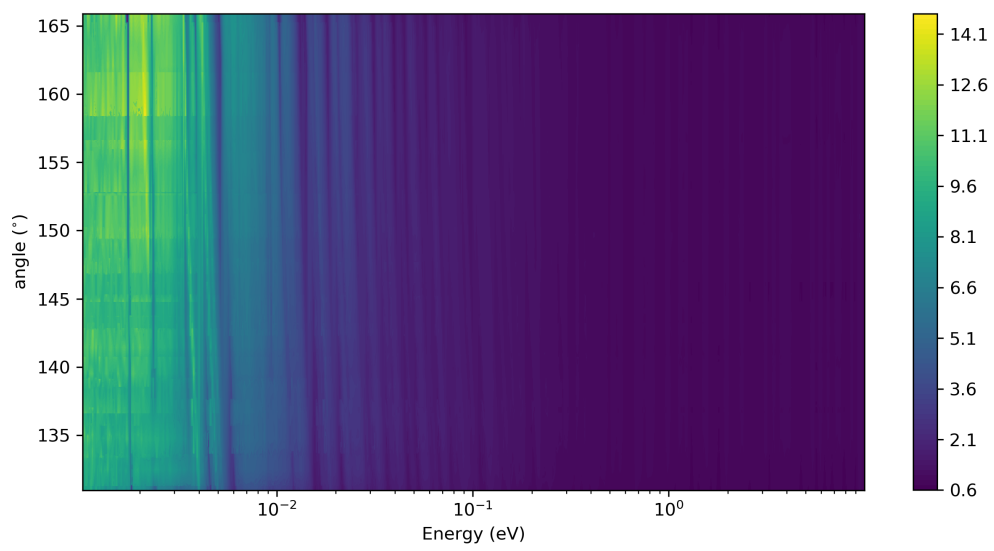

Fig. 8. Energy and angular distribution of the ratio of reflectivities between $\mathrm{MgH}_{2}$ at $20 \mathrm{~K}$ and the nanodiamond sample of $7 \mathrm{~mm}$ thickness for the back-scattering detector banks.

It is expected [19] that the reflectivities of both materials are similar at about $10^{-4} \mathrm{eV}$, and that below that energy the ND will clearly be better. However, as in most current applications we can consider that liquid $\mathrm{H}_{2}$ 


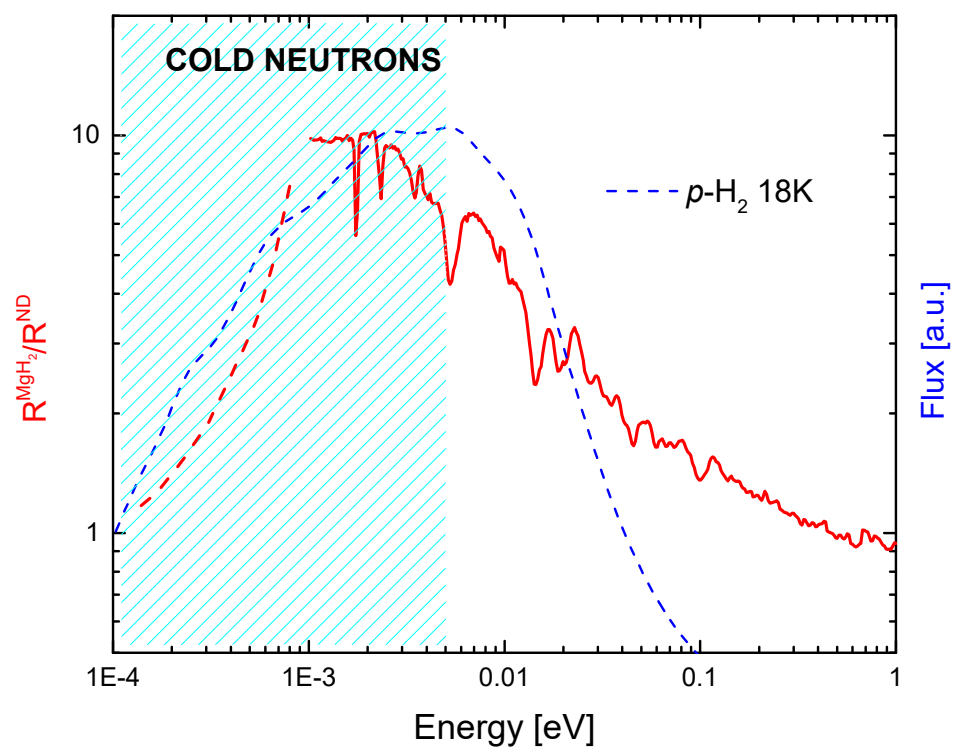

Fig. 9. Typical cold neutron flux emerging from a liquid hydrogen source (blue dashed line) and the ratio of reflectivities between cold $\mathrm{MgH}_{2}$ and ND (red line). The dashed red line represents the expected trend of the reflectivity ratio $(\mathrm{Eq}[5)$ in the cold neutron region.

is the cold neutron moderator and we wish to reflect back into the moderator cell those neutrons leaking in the wrong direction. In Fig. 9 we compare a typical cold neutron flux emerging from a liquid hydrogen source and the ratio of reflectivities between cold Magnesium Hydride and ND determined in this work, extrapolated with the expected trend of the reflectivity ratio in the cold neutron region. Magnesium Hydride is much more efficient than the diamond nanopowder to reflect neutrons produced by a cold source, although the latter should be an excellent coating material to transport cold neutrons on account of its very large quasi specular reflection capacity [20].

\section{Conclusions}

We performed transmission and scattering experiments at room and low temperatures on nanodiamond and $\mathrm{MgH}_{2}$ powder samples, using the VESUVIO instrument at the ISIS pulsed neutron source.

The total cross sections for neutron energies $10^{-3}$ to $10 \mathrm{eV}$ were determined from the transmission measurements on both materials. Especially for the ND samples, a careful in-scattering correction procedure was required, on account of the very large SANS contribution caused by interference effects. We calculated the total cross sections using our scattering kernels and the cross section libraries generated from them, showing an excellent agreement with the corrected experimental total cross sections.

The scattering measurements were used to make relative comparison of the reflecting capacity of $\mathrm{ND}_{\text {and }} \mathrm{MgH}_{2}$. The different sets of detectors were grouped to facilitate the comparison with good statistics. An expression proposed to consider the ratio of reflectivities shows to be adequate to describe the material properties irrespective of the sample thickness employed in the comparison. The results confirm the better performance of $\mathrm{MgH}_{2}$ at low temperatures as compared with nanodiamonds over the "cold neutron reflectivity gap", as we have previously predicted from theoretical considerations.

The actual gain obtained by the use of such reflector will depend on the specific application, and should become more noticeable in systems with high leakage, as in a low dimensional liquid hydrogen source. 


\section{Acknowledgements}

The authors acknowledge the UK Science and Facilities Council for the beam time assigned at the ISIS Neutron Source - Rutherford Appleton Laboratory (VESUVIO, https://doi.org/10.5286/ISIS.E.RB1920136).

\section{References}

[1] E.V. Lychagin, A.Y. Muzychka, V.V. Nesvizhevsky, G.V. Nekhaev, G. Pignol, K.V. Protasov and A.V. Strelkov, Coherent scattering of slow neutrons at nanoparticles in particle physics experiments, Nuclear Instruments and Methods in Physics Research Section A: Accelerators, Spectrometers, Detectors and Associated Equipment 611(2) (2009), 302-305-, Particle Physics with Slow Neutrons. doi:10.1016/j.nima.2009.07.086. https://www.sciencedirect.com/science/article/pii/S0168900209015496

[2] V. Nesvizhevsky, U. Köster, M. Dubois, N. Batisse, L. Frezet, A. Bosak, L. Gines and O. Williams, Fluorinated nanodiamonds as unique neutron reflector, Carbon 130 (2018), 799-805-. doi:10.1016/j.carbon.2018.01.086. https://www.sciencedirect.com/science/article/pii/ S0008622318300952

[3] J.R. Granada, J. Marquez Damian and C. Helman, Studies on Reflector Materials for Cold Neutrons, EPJ Web of Conference 231(04002) (2020), 6 .

[4] V.V. Nesvizhevsky, Interaction of neutrons with nanoparticles, Physics of Atomic Nuclei 65(3) (2002), 400. doi:10.1134/1.1465480.

[5] V.V. Nesvizhevsky, M. Dubois, P. Gutfreund, E.V. Lychagin, A.Y. Nezvanov and K.N. Zhernenkov, Effect of nanodiamond fluorination on the efficiency of quasispecular reflection of cold neutrons, Phys. Rev. A 97 (2018), 023629. doi:10.1103/PhysRevA.97.023629.

[6] V.A. Artem'ev, A.Y. Nezvanov and V.V. Nesvizhevsky, Precise calculations in simulations of the interaction of low energy neutrons with nano-dispersed media, Crystallography Reports 61(1) (2016), 84-88-. doi:10.1134/S1063774516010028.

[7] M. Teshigawara, Y. Tsuchikawa, G. Ichikawa, S. Takata, K. Mishima, M. Harada, M. Ooi, Y. Kawamura, T. Kai, S. Ohira-Kawamura, K. Nakajima, Y. Ikeda and Y. Kiyanagi, Measurement of neutron scattering cross section of nano-diamond with particle diameter of approximately $5 \mathrm{~nm}$ in energy range of $0.2 \mathrm{meV}$ to $100 \mathrm{meV}$, Nuclear Instruments and Methods in Physics Research Section A: Accelerators, Spectrometers, Detectors and Associated Equipment 929 (2019), 113-120-. doi:10.1016/j.nima.2019.03.038. https://www.sciencedirect.com/science/article/pii/S0168900219303456

[8] P. Giannozzi, O. Andreussi, T. Brumme, O. Bunau, M.B. Nardelli, M. Calandra, R. Car, C. Cavazzoni, D. Ceresoli, M. Cococcioni, N. Colonna, I. Carnimeo, A.D. Corso, S. de Gironcoli, P. Delugas, R.A. DiStasio, A. Ferretti, A. Floris, G. Fratesi, G. Fugallo, R. Gebauer, U. Gerstmann, F. Giustino, T. Gorni, J. Jia, M. Kawamura, H.-Y. Ko, A. Kokalj, E. Küçükbenli, M. Lazzeri, M. Marsili, N. Marzari, F. Mauri, N.L. Nguyen, H.-V. Nguyen, A. Otero-de-la-Roza, L. Paulatto, S. Poncé, D. Rocca, R. Sabatini, B. Santra, M. Schlipf, A.P. Seitsonen, A. Smogunov, I. Timrov, T. Thonhauser, P. Umari, N. Vast, X. Wu and S. Baroni, Advanced capabilities for materials modelling with Quantum ESPRESSO, Journal of Physics: Condensed Matter 29(46) (2017), 465901. doi:10.1088/1361-648x/aa8f79.

[9] A.C. Victor, Heat Capacity of Diamond at High Temperatures, The Journal of Chemical Physics 36(7) (1962), 1903-1911-. doi:10.1063/1.1701288

[10] R. Macfarlane, D.W. Muir, R. Boicourt, A.C. Kahler III and J.L. Conlin, The NJOY nuclear data processing system, version 2016, Technical Report, Los Alamos National Lab.(LANL), Los Alamos, NM (United States), 2017.

[11] X.-X. Cai and T. Kittelmann, NCrystal: A library for thermal neutron transport, Computer Physics Communications 246 (2020), 106851.

[12] G. Beaucage, Small-Angle Scattering from Polymeric Mass Fractals of Arbitrary Mass-Fractal Dimension, Journal of Applied Crystallography 29(2) (1996), 134-146-. doi:10.1107/S0021889895011605.

[13] L.A. Rodríguez Palomino, J. Dawidowski, C. Helman, J.I. Márquez Damián, G. Romanelli, M. Krzystyniak, S. Rudić and G.J. Cuello, Determination of the scattering cross section of calcium using the VESUVIO spectrometer, Nuclear Instruments and Methods in Physics Research Section A: Accelerators, Spectrometers, Detectors and Associated Equipment 927 (2019), 443-450. doi:https://doi.org/10.1016/j.nima.2019.02.072. https://www.sciencedirect.com/science/article/pii/S0168900219302682

[14] G. Romanelli, M. Krzystyniak, R. Senesi, D. Raspino, J. Boxall, D. Pooley, S. Moorby, E. Schooneveld, N.J. Rhodes, C. Andreani and F. Fernandez-Alonso, Characterisation of the incident beam and current diffraction capabilities on the VESUVIO spectrometer, Measurement Science and Technology 28(9) (2017), 095501. doi:10.1088/1361-6501/aa7c2a.

[15] J. Robledo, J. Dawidowski, J. Márquez Damián, G. Škoro, C. Bovo and G. Romanelli, Measurement of neutron total cross sections at the VESUVIO spectrometer, Nuclear Instruments and Methods in Physics Research Section A: Accelerators, Spectrometers, Detectors and Associated Equipment 971 (2020), 164096.

[16] A. Di Giulio, M. Zanetti, G. Romanelli, M. Krzystyniak, R. Senesi and F. Fernandez-Alonso, A McStas simulation of the incident neutron beam on the VESUVIO spectrometer, Journal of Physics: Conference Series 1055(1) (2018), 012014.

[17] S.C. Capelli and G. Romanelli, An effective hydrogen scattering cross section for time-of-flight neutron experiments with simple organic molecules, Journal of Applied Crystallography 52(5) (2019), 1233-1237. doi:10.1107/S1600576719011592. 
[18] O. Arnold, J.C. Bilheux, J.M. Borreguero, A. Buts, S.I. Campbell, L. Chapon, M. Doucet, N. Draper, R.F. Leal, M.A. Gigg, V.E. Lynch, A. Markvardsen, D.J. Mikkelson, R.L. Mikkelson, R. Miller, K. Palmen, P. Parker, G. Passos, T.G. Perring, P.F. Peterson, S. Ren, M.A. Reuter, A.T. Savici, J.W. Taylor, R.J. Taylor, R. Tolchenov, W. Zhou and J. Zikovsky, Nuclear Instruments and Methods in Physics Research A Mantid - Data analysis and visualization package for neutron scattering and $\mu$ SR experiments, Nuclear Inst. and Methods in Physics Research, A 764 (2014), 156-166. doi:10.1016/j.nima.2014.07.029.

[19] Y. Charles, D. Kiselev, A. Takibayev, M. Wohlmuther and L. Zanini, The 1st Workshop on Efficient Neutron Sources ENS2019, Zenodo, 2020. doi:10.5281/zenodo.4432456.

[20] M. Jamalipour, L. Zanini and G. Gorini, Directional reflection of cold neutrons using nanodiamond particles for compact neutron sources, in: EPJ Web of Conferences, Vol. 231, EDP Sciences, 2020, p. 04003. 